\title{
Tactile letter recognition under different modes of stimulus presentation*
}

\author{
JACK M. LOOMIS $†$ \\ Smith-Kettlewell Institute and Department of Visual Sciences, Pacific Medical Center \\ San Francisco, California 94115
}

\begin{abstract}
Block capital letters were displayed to experienced and inexperienced Ss, using a $20 \times 20$ matrix of vibratory tactors placed against the back. In two separate experiments, a total of five modes of stimulus presentation, three of them employing a linear scanning slit, were studied. The poorest method, stationary flashing of the letter, allows performance that is well above chance, implying that a purely spatial presentation does convey information. Performance is improved when the letter is moved horizontally across the display. The best performance is achieved when the amount of simultaneous stimulation is limited by using a linear scanning slit. In one method, the letter moves behind a stationary slit, with the result that its horizontal dimension is portrayed only in time. In the other two methods, the scanning slit moves across the stationary letter, portraying the letter both in time and in space. The results of all five display modes indicate that $\mathrm{Ss}$ can use whichever representation, spatial or temporal, is available, although patternings which most closely approximate sequential tracing by a single moving point lead to the highest recognition accuracy. We interpret these results in terms of the limited spatial resolution of the cutaneous sense. While the perception of a letter presented in either full-field condition is limited by the spatial resolution, the best measure being the two-point limen, the perception of a letter traced sequentially is limited by the localization acuity of the cutaneous sense, the best measure being the "error of localization," which is known to be considerably smaller than the two-point limen. Inasmuch as the slit methods of presentation are a compromise between simultaneous and sequential display, letter-recognition accuracy is better with slit presentation than with the corresponding full-field mode of display.
\end{abstract}

Because the skin, like the retina, is a spatially extended receptive surface, it has more than once been considered as an alternative channel for transmitting spatial patterns which might otherwise be handled by the visual system. The qustion that arises is how to display spatial patterns on the skin to best exploit its processing capabilities. We are all familiar with "finger writing" on the back as a way of communicating patterns; although moving a single point in time and space permits high recognition accuracy for simple patterns, it suffers from the disadvantages of burdening the observer's memory and requiring fairly elaborate preprocessing when used with a video input.

*This investigation was supported by the Department of Health, Education, and Welfare, Grant 14-P-55282 from the Social and Rehabilitation Service, and by National Institutes of Health Postdoctoral Fellowship F02 MH51765-01 PS to the author. A report of some of these results was given in a paper delivered at the Western section meeting of the Association for Research in Vision and Ophthalmology held in Vancouver, B.C., in September 1972. The advice of Lawrence $A$. Scadden during the planning of this research is gratefully acknowledged. The author also wishes to thank Paul Bach-y-Rita, Ken Nakayama, Jon C. Taenzer, and Benjamin W. White for their comments on the manuscript.

†Present address: Department of Psychology, University of California, Santa Barbara, California 93106.
A more compatible but much less informative method of presentation is to impress the spatial form all at one time onto the surface of the skin. In these experiments we have considered several compromise modes of presentation that require relatively simple preprocessing of the spatial form. These modes of presentation differ in how they represent the form spatially and temporally, and thus allow some insight into the way the cutaneous sense handles information.

We have used block letters as forms in these experiments, not so much for the reason that we are interested in letter recognition per se but because the alphabet is a highly diverse, yet familiar, set of forms.

\section{EXPERIMENT I}

\section{Method}

Apparatus. The primary piece of equipment used in these experiments was the 400 -point vibrotactile version of the vision substitution system developed by Paul Bach-y-Rita and Carter C. Collins, and described in detail elsewhere (Collins, 1970; Scadden, 1971; Bach-y-Rita, 1972).

Briefly, the video signal from a television camera drives a $20 \times 20$ matrix of solenoid vibrators; if the light flux falling on a given camera element exceeds an adjustable threshold value, the corresponding tactor is activated. Since a stimulator is either "on" or "off," the tactile image corresponding to any optical image has, as it were, two "brightness" levels. To reduce problems with noise, we typically use high-contrast visual displays. 
The factors vibrate at $60 \mathrm{~Hz}$ when activated, and have an activation rise time of several milliseconds and a decay time of one or two cycles (16-33 msec); the power supply voltage was set in these experiments so that each stimulator was operating at $10 \mathrm{~dB}$ above threshold $(50 \mathrm{~mW})$. With interstimulator spacing of $12 \mathrm{~mm}$, both vertically and horizontally, the full $20 \times 20$ matrix covers a square area on the back of $23 \mathrm{~cm}$ on a side. For most Ss, 16 or more of the columns and all of the rows make good contact with the surface of the back.

In its normal configuration, the system employs a freely moving camera mounted on a boom which permits the $S$ to manually scan the optic array. Inasmuch as this mode of operation does not allow an experimental separation of the reafferent information available through manual control of the camera from the information that is extracted from the tactile display, we employed a stationary camera to study exclusively the registration characteristics of the cutaneous sense.

In order to present letters to the stationary camera in the various modes desired, we constructed a simple apparatus. It consisted of a diffuising screen for back-illumination of the stimuli, a moving belt with a slot into which the letter stimuli or scanning slits could be inserted, and a stationary slot, also for the letters or slits, just in front of the screen. The moving slot passed just in front of the stationary slot so that in either slot the letters subtended the same visual angle at the camera. The stimuli were 26 cardboard squares out of which capital block letters had been cut (glass slides glued onto the cards were used to retain the internal sections of letters like $\mathrm{A}, \mathrm{B}, \mathrm{D}, \mathrm{O}$, etc.). When both a slit and a letter were inserted into the appropriate slots, the light that reached the camera was that which passed through both of the cards.

Stimuli. In terms of the stimulator matrix, all the capital block letters except I were 18 tactors high, 13 wide, and 2 in stroke width. Four modes of presentation were studied in the first experiment (Fig. 1): in the full-field, stationary-letter mode, letters were inserted into the stationary slot and exposed for $1.5 \mathrm{sec}$; in the moving-slit stationary-letter mode, a vertical scanning slit was also inserted into the moving belt and was moved from left to right at 16 columns/sec, giving a total exposure time of a little over $1 \mathrm{sec}$; in the third method, full field, moving letter, the letter was inserted

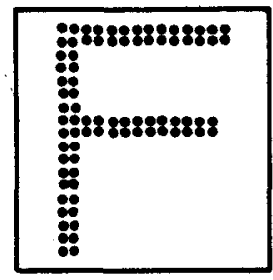

Full Field, Stationary Letter

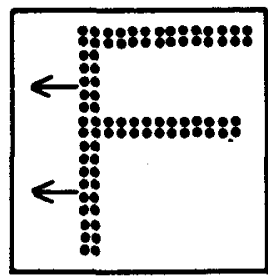

Full Field, Moving Letter

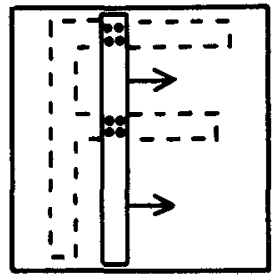

Moving Slit, Stationary Letter
Fig. 1. Representation of the four modes of stimulus presentation used in Experiment I. See text for details. into the moving slot and was transported from right to left at 16 columns/sec, giving a total exposure of 1.7 to $2.0 \mathrm{sec}$ (depending upon the extent of the display in contact with the S's back); finally, in the stationary-slit, moving-letter method, the vertical slit was positioned in the stationary slot and the letter moved by from right to left at 16 columns/sec, again giving an exposure of just over $1 \mathrm{sec}$. Observe that in each of the last three methods, it is the leftmost part of the letter which was initially exposed. The total exposure times necessarily differed since the rate of movement across the display was held constant; the longer exposure times favor the two full-field methods, which later proved to give poorer letter-recognition performance.

Subjects. A total of seven Ss were run in these two experiments. Three of them-L.S., M.C., and W.G.-were blind and had each had well over $100 \mathrm{~h}$ of practice in recognizing objects and letters with the system in its normal mode of operation, namely, active scanning with the camera. Typically, in that mode, the $S$ chooses to set the zoom lever on the camera such that only a fraction (one-eighth to one-half) of a letter fills the display; by actively scanning for features, the $S$ is able to achieve near perfect recognition accuracy by taking as much time as needed to reach a decision (latencies of 1 to $9 \mathrm{sec}-\mathrm{L}$. A. Scadden, personal communication). The method which most resembles this scanning technique is the full-field, moving-letter method.

The remaining four Ss were all sighted and had had no prior experience with the system. They were, of course, blindfolded at all times. All seven Ss were in their 20 s or early 30 s; two of the sighted Ss were women.

Procedure. Each 2-h session consisted of 208 presentations, twice of each letter of the alphabet under each of the four modes of presentation. Every 13 trials, the presentation mode was switched according to a random schedule. On each trial, Ss were given a warning signal and then the letter presentation, after which they responded. Ss were given as much time to respond as they needed; latencies generally ranged between 0.5 and $5 \mathrm{sec}$. If their first response was incorrect, they responded with a second guess, after which they were informed of the correct response. The second-guess data have not been included in the analysis, as they proved not to add anything beyond the analysis of the first responses.

Each of the experienced Ss was run a total of three 2-h sessions, with all of the data figuring into the analysis; the inexperienced Ss, in contrast, were each run six sessions, with the data from only the last three sessions being included in the analysis; by the fourth session, their performances had leveled off, though perhaps at temporary plateaus well below levels which could be expected of much longer training. Thus, the overall analysis is based on 624 letter presentations for each of seven Ss or 1,092 trials for each of the four methods of presentation.

\section{Results}

The first-guess recognition accuracies for each $S$ as a function of mode of presentation are shown in Fig. 2. Perhaps the most striking feature is the extremely wide range of performances by the different Ss, with the three blind, experienced Ss exhibiting the best performance. Not surprisingly, a mixed model analysis of variance reveals $S$ differences to be significant at the .01 level $(F=194.2$, df $=6 / 56)$. S W.G., incidentally, has consistently outperformed all other $\mathrm{Ss}$, experienced or not, on a wide variety of tasks with the system; these data indicate that his superior performance is at least partly attributable to a greater capability of registering and processing information in the tactile display.

Of greater interest is the comparison of methods. In Fig. 2, one can observe for each $S$ a downward trend of performance from left to right. The Friedman test (Hays, 1963, p. 640) indicates a consistency of the 


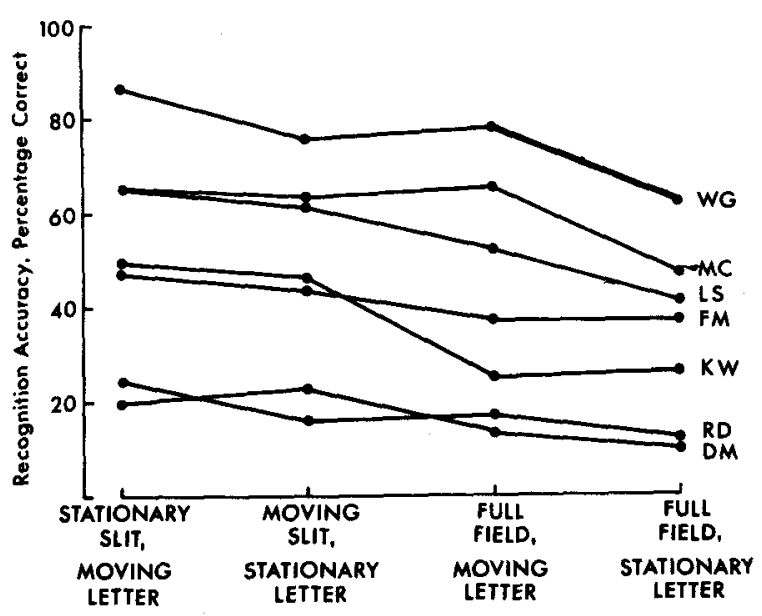

Fig. 2. First-response recognition accuracy for each of the Ss under each of the four modes of stimulus presentation.

orderings of the four methods across $S s(p<.01)$. The average performance for each method is shown in Fig. 3. A mixed model analysis of variance (Hays, 1963, p. 439) reveals a significant methods effect at the .01 level $(\mathrm{F}=16.6$, $\mathrm{df}=3 / 18)$ and a significant Ss by Methods interaction at the .05 level $(F=2.36$, $\mathrm{df}=18 / 56$ ). The analysis assumed all four methods as a single factor rather than as two (slit/full field and moving/stationary) because as factors they are not truly independent, inasmuch as the type of slit presentation depends upon whether the letter was moving or stationary. Further comparison of pairs of methods using the Student-Newman-Keuls procedure (Winer, 1962, p. 77) indicates that all methods differ significantly from each other at the .05 level except for the two slit methods. Of primary interest will be the following comparisons, all significant at the .01 level: full field, stationary letter vs full field, moving letter; stationary slit, moving letter vs full field, moving letter; and moving slit, stationary letter vs full field, stationary letter.

The confusion matrices for each of the four methods are shown in Figs. 4-7. The cell values represent the total number of trials (summed over Ss) on which the letter indicated by the column was given as a response to the stimulus letter indicated by the row. Each letter was presented a total of 42 times under each method.

\section{Discussion}

The first point of interest is that the full-field, stationary-letter method yields an overall recognition accuracy $(34 \%)$ that is well above chance $(5.2 \%)$, ${ }^{1}$ with W.G. achieving a modestly high $62 \%$, indicating that some information is extracted from a purely spatial presentation. Examination of the confusion errors for this method (Fig. 4) suggests that letters are discriminated on the basis of global shape rather than fine detail; in the framework of spatial frequency analysis, it appears that letters that differ in their low spatial frequency components are more readily discriminable than those differing in the high spatial frequencies. The responses to $W$ illustrate this point; what $W$ has in common with $B, N$, and $O$ is the fact that it covers the $13 \times 18$ letter space more or less uniformly. Other response patterns that are consonant with the idea that the higher spatial frequencies are lost in the cutaneous processing are the confusions between $B, D, G, O, Q$, and $S$, between $P$ and $F$, and between $Y$ and $T$. It should be emphasized that the tactile display itself is not limiting; the finest differentiating features, such as the extra stroke that distinguishes $Q$ from $O$ are clearly visible in the 400 -point visual monitor.

The next point of interest is that moving the entire letter (full field, moving letter) across the display (at $19.2 \mathrm{~cm} / \mathrm{sec}$ ) improves performance over that of the stationary presentation, especially for the experienced observers (Fig. 2). The same result has been observed in studies on pattern recognition with the finger by Austin and Sleight (1952), Bliss and Crane (1965), Linvill and Bliss (1966), and Bliss, Crane, and Link (1966).

Of greatest interest is the finding that each of the two slit methods is superior to the corresponding full-field method $(p<.01)$, with all seven of the Ss performing better with the slit mode for stationary letters and six out of the seven, for moving letters. We interpret these differences in performance in terms of the limited resolution of the cutaneous sense.

The explanation we propose hinges upon the distinction between spatial resolution and localization acuity, the former relating to the ability of a system to resolve two points and the latter to its ability to localize a single point. In the terms of linear systems analysis, spatial resolution is defined as the highest spatial frequency that is transmitted by the system, here the cutaneous sense. The conventional measure of tactile resolution is the two-point limen, which is presumably the inverse of the upper limiting spatial

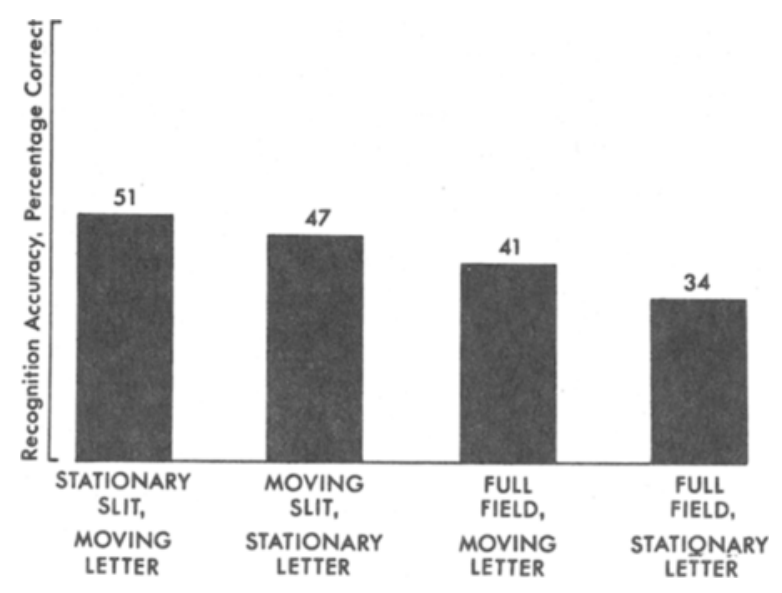

Fig. 3. Average recognition accuracy as a function of method of presentation. 


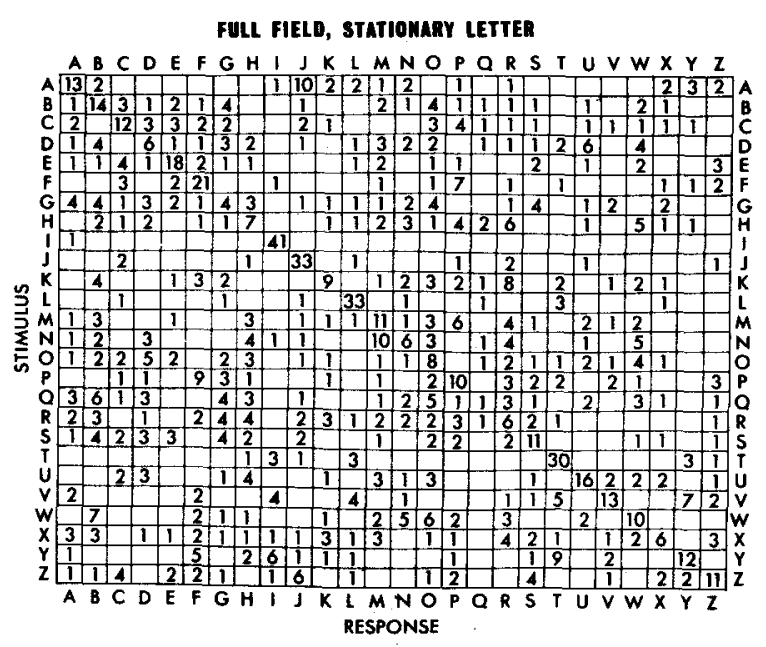

Fig. 4. Confusion matrix for the full-field, stationary-letter mode.

frequency. For the back, it is about $18 \mathrm{~mm}$ with our type of vibrotactile stimulation (Eskildsen, Morris, Collins, \& Bach-y-Rita, 1969). Localization acuity, on the other hand, is presumably determined by the sensitivity of the sensory system to phase shifts in one or more of the component spatial frequencies, perhaps even the lowest ones. The conventional measure of localization acuity for the cutaneous sense is the "error of localization," which has been found to be considerably smaller than the two-point limen, body locus and type of stimulation being equal (Boring, 1930, 1942; Dallenbach, 1932; Jenkins \& Stone, 1941; Weinstein, 1968; Zigler, 1935).

It is a fundamental point that localization acuity in principle is not limited by spatial resolution, although in fact the two measures do covary across body loci (Weinstein, 1968), probably reflecting the common neural organization. The reasoning behind the above statement goes as follows. A single point input to any

MOVIME SLIT, STATIOMARY LETTER

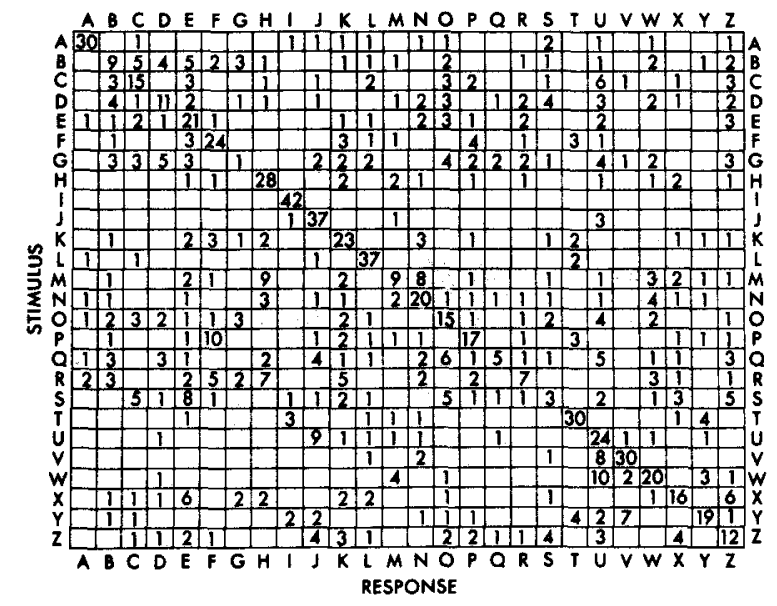

Fig. 6. Confusion matrix for the moving-slit, stationary-letter mode.

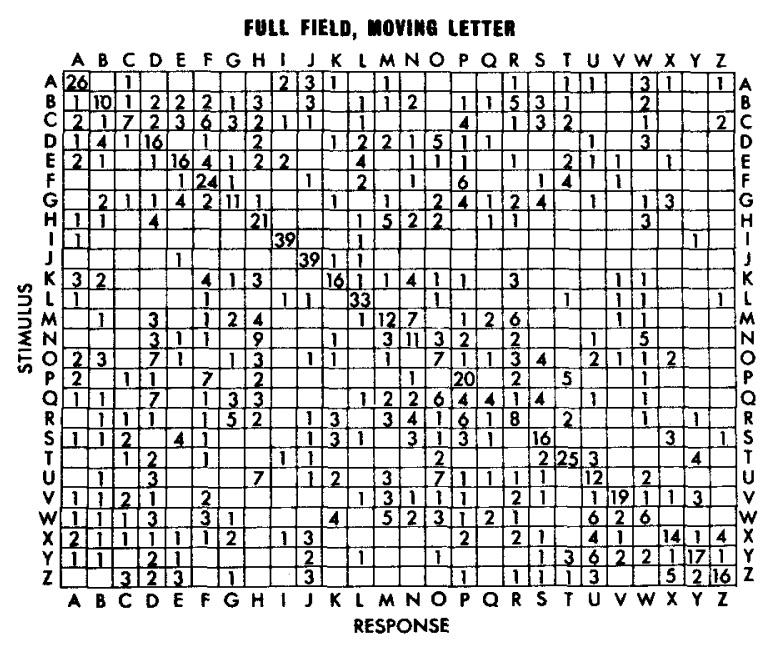

Fig. 5. Confusion matrix for the full-field, moving-letter mode.

spatial system, conceived as a spatial filter, is represented in the spatial frequency domain by all frequencies, they being in phase at the position of the target. Wherever the target moves, naturally, these spatial frequency components shift so that they remain in phase at the target position. Even if the higher spatial frequencies (those limiting resolution) are lost in the transfer through the system, the phase information in the lower spatial frequencies carries the position information. Thus, if the sensory channel is sensitive to phase shifts in the lower spatial frequencies, it can localize a point target with greater precision than it can resolve two points. This argument, expressed physiologically in terms of receptive field organization of the somatosensory system, could explain why the error of localization is so much smaller than the two-point limen.

The relevance of this argument can now be stated. When a letter is presented in either of the full-field

\section{STATIOMARY SLIT, MOVIME LETTER}

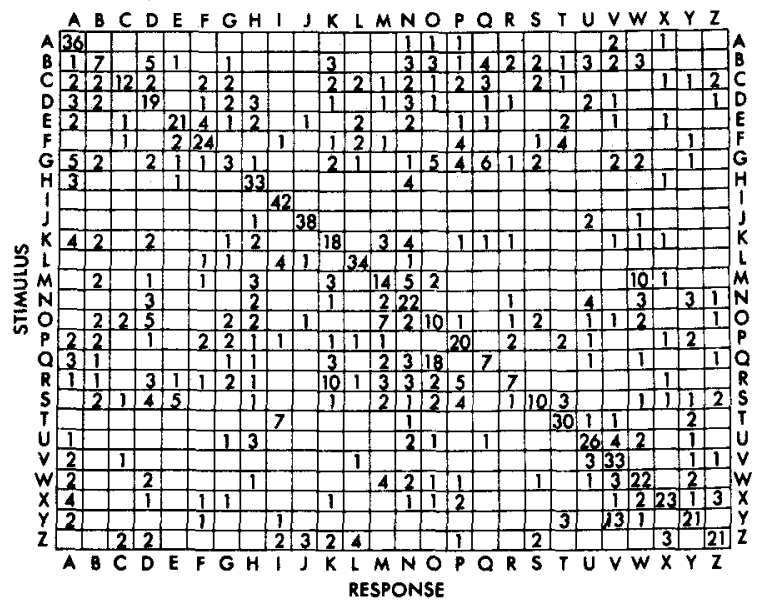

Fig. 7. Confusion matrix for the stationary-slit, moving-letter mode. 
modes, the identity of the letter is carried by the various spatial frequency components. Some letter discriminations can be made solely on the basis of the lower spatial frequencies (like $L$ from $T$ and $I$ from $\mathrm{O})$, whereas finer discriminations require registration of higher frequencies (like $P$ from $F$ and $O$ from $D$ ). Because the confusion errors in both full-field methods roughly conform to this pattern, it is reasonable to suppose that performance is limited by resolution. Although the back has poorer resolution than other body loci (Weinstein, 1968), probably reflecting the neural organization of the cutaneous sense, the same general pattern of confusion would be expected for other body loci with letters of appropriate size.

If, however, a letter were traced out in time by a single moving point, as in finger writing, one would expect letter recognition to be limited more by localization acuity than by spatial resolution. This follows because phase information in the lower spatial frequencies, varying as a function of time, would carry the identity of the letter. That sequential tracing of a letter does lead to higher recognition accuracy than full-field presentation has been shown with the TVSS (Beauchamp, Matheson, \& Scadden, 1971). This can also be demonstrated with the visual sense by observing letters through a low-pass spatial filter (e.g., a strong spherical lens or a diffusing screen) that are presented either full field or sequentially, as when traced by a moving point of light. Informal investigation here indicates that letters which in the full-field condition appear only as a large blur can be recognized in the sequential presentation mode.

Although this experiment did not employ a sequential tracing mode, the two slit methods are a compromise between sequential tracing and full-field presentation, inasmuch as each vertical volumn is presented separately in time (and in space in the moving-slit, stationary-letter mode), with the information in that column being simultaneous. Presenting each vertical slice of the letter in time means that it need not be spatially resolved from the other columns which precede or follow it in time. When a system behaves as a low-pass spatial filter, as the cutaneous sense does, the input pattern is effectively spread out (by convoluting the input pattern with the "point spread function" of the system) so that the highest spatial frequencies are completely lost and the lower frequencies are reduced in effective contrast. Although the stimulation in each column does continue to spread horizontally in the two slit conditions, the different columns of each letter no longer interfere with one another by virtue of their appearing at different points in time; thus the spatial information within each column is reduced in contrast only by the vertically spreading stimulation.

Further support for this idea comes from an examination of the confusion matrices (Figs. 4-7). From our earlier argument, it is plausible that when only a single point of stimulation is present within a vertical column, it is accurately perceived in location. In the moving-slit, stationary-letter condition, the letters $\mathrm{V}$ and $\mathrm{W}$ are actually traced out sequentially. In the stationary-slit, moving-letter mode, the horizontal dimension is collapsed onto the single active column (actually two adjacent columns in the display) so that these two letters are represented by a single point moving vertically within the column. In both conditions, $\mathrm{V}$ and $\mathrm{W}$ show substantial gains in recognition accuracy over the corresponding full-field conditions. However, these are not the only letters that would be expected to improve, for letters that activate either a full vertical column (corresponding to a vertical stroke) or a single point within the column $(\mathrm{H}, \mathrm{I}, \mathrm{J}, \mathrm{L}, \mathrm{M}, \mathrm{N}, \mathrm{T}, \mathrm{U}$, and $\mathrm{Y})$ should be recognized as easily. In addition, the letter $A$ is characterized by a single moving point through half of its presentation, which, because of its unique patterning, makes it readily identifiable. All other letters activate at least two vertical locations during the horizontal sweep of the letter, so that the vertically spreading stimulation makes them most difficult to recognize. Comparing the relative gain in performance from each full-field condition to the corresponding slit condition for the two letter subsets (A, H, I, J, L, M, N, T, U, V, W, Y) and $(B, C, D, E, F, G, K, O, P, Q, R, S, X, Z)$, we found a significantly larger gain for the former subset both with stationary letters $(\mathrm{t}=2.08$, $\mathrm{df}=24$, $\mathrm{p}<.025)$ and with moving letters $(\mathrm{t}=3.12, \mathrm{df}=24$, $\mathrm{p}<.005)$. These results would be even more decisive if the letters I, J, and $L$ had been exluded from the analysis because of their already high recognizability in the full-field conditions. This pattern of gains (and losses) in recognition accuracy in going from full-field to a slit presentation is consistent for both moving and stationary letters, as indicated by the positive correlation of the gain scores $(r=.77, p<.001)$.

In summary, we conclude that letters are more recognizable when scanned by a vertical slit than when presented complete, all at one time, because the deleterious effect of horizontally spreading stimulation is eliminated with the column-by-column presentation. Moreover, letters which activate each vertical column during the sweep in a manner most resembling sequential tracing exhibit the largest gains in recognition accuracy, lending support to the argument that this form of presentation is less subject to the limited resolution of the cutaneous sense.

It is interesting to note that even though the stationary-slit, moving-letter mode collapses the spatiotemporal patterning of the moving-slit, stationary-letter mode onto essentially a single column, performance suffers not in the least, with six out of the seven Ss actually performing slightly better (t not significant). This demonstrates that $S$ s are quite able to integrate the activity of a single vertical column over time to reconstruct the horizontal dimension. This same conclusion was drawn by 
Dinnerstein and Wolfe (1962) in a study of letter recognition in which embossed letters were sensed by the finger through a vertical slit.

Another point worth noting is that although the full-field, moving-letter mode is essentially 10 adjacent replications of the stationary-slit, movingletter mode, the latter mode gives superior performance $(p<.01)$, with six out of the seven Ss favoring the latter mode. This means that the same time-varying information in two columns is less easily sensed when these two columns are part of a larger display than when they alone are active. In the light of our interpretation in terms of horizontal spreading, these results pose no real paradox.

However, it should be mentioned that there are some results that seem to contradict this finding. Several word-reading studies (Linvill \& Bliss, 1966; Taenzer, 1971, 1972; Baer \& Hill, 1972) with the Optacon, a vibrotactile reading aid for the blind, have found that one- or two-column displays (on the finger) give substantially poorer performance than larger displays. To explain the difference in results, one could cite a number of differences between the Optacon and the TVSS, such as body locus, display size, and frequency of stimulation $(250$ vs $60 \mathrm{~Hz})$; however, the most plausible reason has been suggested by Taenzer (personal communication). When Optacon users are inexperienced, they perform better with one- or two-column displays. As they become more proficient at reading, they increase their scanning rates beyond the point where they can use the temporal modulations of a single column. They are able to do so because of their extensive practice in recognizing letters in terms of global shape and because of their proficiency in using contextual information; thus, experienced users perform better with larger displays at high reading rates.

\section{EXPERIMENT II}

During the running of the first experiment, the impression developed that it was the cognitive simplicity of the spatiotemporal patterns produced by scanning with a vertical slit that permitted the $S$ to integrate these patterns with relative ease. It therefore seemed unlikely that Ss would have any success in recognizing the spatiotemporal representations of letters when a diagonal scanning slit was used in place of the vertical one in the moving-slit, stationary-letter mode. This expectation was totally unsupported by the data below.

\section{Method}

After completing the first experiment, each $\mathrm{S}$ participated in two more sessions, each of $2 \mathrm{~h}$ length. Half of the trials of each session were run using the moving-slit, stationary-letter mode of Experiment $\mathbf{I}$. The rest of the trials were run using a modified moving-slit, stationary-letter mode in which the scanning slit was angled at $45 \mathrm{deg}$ (Fig. 8). It can be seen from the figure that the temporal patterning is more complex than with the vertical slit, even though exactly the same spatial locations are stimulated over
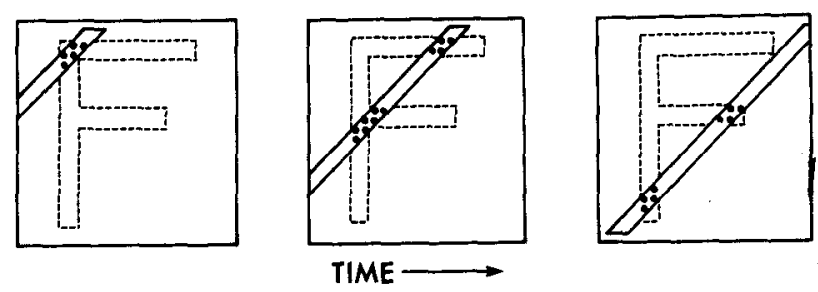

Fig. 8. Representation of the sequence of stimulation in the diagonal slit version of the moving-slit, stationary-letter mode of Experiment II.

the presentation interval. Because the slit moved with the same horizontal velocity in the two conditions, the 13-column-wide letters were displayed in just under $2 \mathrm{sec}$ in the diagonal slit mode and in about $0.8 \mathrm{sec}$ in the other; this follows from the fact that the letters are longer than they are wide and the fact that a diagonal slit would have to traverse a longer distance even if the letter were square.

As before, the mode of presentation was changed every 13 trials, giving a total of 104 presentations for each of the methods per session. The rest of the procedure was identical with that of the previous experiment.

\section{Results}

The recognition accuracies of the first-guess responses as a function of day and mode of presentation are shown in Fig. 9. Although the differences between the two modes are significant at the .05 level for both the first and second sessions, what is important is that Ss do surprisingly well from the beginning, with the indication that they may eventually attain the performance levels of the vertical mode.

To give some idea of the importance of temporal patterning, the first-guess recognition accuracies for the first day as a function of letter and mode of presentation are shown in Fig. 10. In addition to showing the overall loss in recognizability with diagonal scanning, the table indicates that the losses in recognizability are selective. In the second session, these letter-specific differences remained, but to a lesser extent, as Ss gained experience with the diagonal slit method.
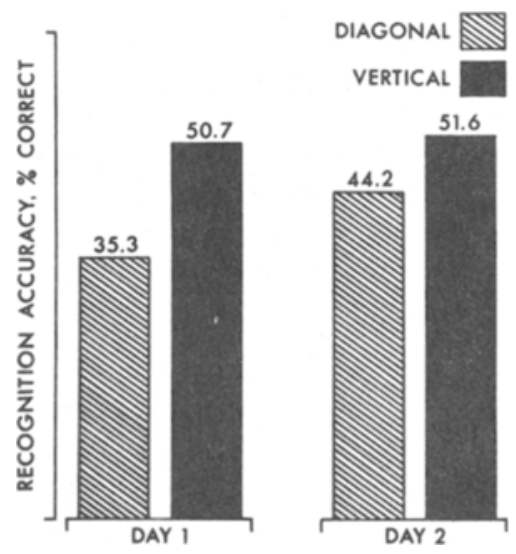

Fig. 9. Average recognition accuracy as a function of mode of presentation and session. 
A B CDEFGH J K L MNOPQRSTUVWXYZ

Fig. 10. Recognition accuracy [percent correct] for each letter as a function of mode of presentation. These are averages over all $S s$ for the first session.

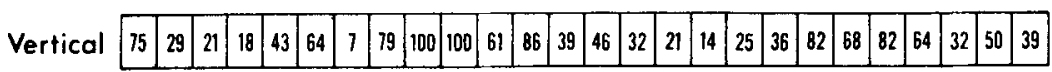

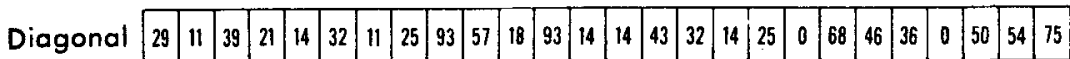

\section{Discussion}

Even though diagonal scanning produces what appear on the visual monitor as very complicated spatiotemporal patterns, these results demonstrate that the method presents no real difficulty for cutaneous processing. Apparently, Ss are able to register these patterns in spite of their complexity, perhaps aided by a kind of tactile memory storage (Bliss, Crane, Mansfield, \& Townsend, 1966; Hill \& Bliss, 1968).

Using the dichotomous classification of the letters from Experiment I, with the more difficult subset (B, $\mathrm{C}, \mathrm{D}, \mathrm{E}, \mathrm{F}, \mathrm{G}, \mathrm{K}, \mathrm{O}, \mathrm{P}, \mathrm{Q}, \mathrm{R}, \mathrm{S}, \mathrm{X}, \mathrm{Z}$ ) being those letters which activate two or more points in each vertical section, a highly significant difference in recognition accuracy $(\mathrm{t}=5.77, \mathrm{df}=24, \mathrm{p}<.001)$ was obtained with vertical scanning. With diagonal scanning, however, a nonsignificant difference $(t=$ 1.69 , $\mathrm{df}=24$ ) was found for the same dichotomy, indicating that the pattern of recognition accuracies reflects the interaction of the vertical slit with the particular letters rather than the Ss' letter-guessing tendencies. Furthermore, the diagonal scanning mode presents all but three of the letters with several points active during most of the presentation; the three exceptions are I, L, and $\mathrm{Z}$, which are sequentially traced by a single moving point. That these three letters have the three highest recognition scores for the diagonal slit condition supports the notion that letters which are sequentially traced are most easily recognized. Further support comes from examination of the scores for $A$ and $W$, which are both very distinctive in the vertical slit condition but are rarely identified with the diagonal scan. While $W$ is sequentially traced by the vertical slit, in the diagonal slit condition it appears as three points moving down the display.

These results and those of Experiment I strongly indicate that when a spatial pattern is sequentially traced or scanned by a linear slit, which is somewhat like one-dimensional tracing, it is more recognizable than when presented in totality all at one time. We have argued in the previous discussion that this is consistent with a system which is limited in spatial resolution.

\section{REFERENCES}

Austin, T, R., \& SLEIGHT, R. B. Accuracy of tactual discrimination of letters, numerals and geometric forms. Journal of Experimental Psychology; 1952, 43, 239-247.
BACH-Y-RITA, P. Brain mechanisms in sensory substitution. New York: Academic Press, 1972.

BaER, J. A., \& HurL, J. W. Optical-to-tactile image conversion for the blind. Final Report, Stanford Research Institute, Contract SRS 70-42 and Grant 14-P-55296/9-02, Menlo Park, California, 1972.

Beauchamp, K. L., Matheson, D. W., \& Scadden, L. A. Effect of stimulus-change method on tactile-image recognition. Perception \& Motor Skills, 1971, 33, 1067-1070.

Bliss, J. C., \& CrANE, H. D. Experiments in tactual perception. NASA Contractor Report NASA CR-322, November 1965, prepared by Stanford Research Institute, Menlo Park, California, under Contrast NAS 2-1679.

Bliss, J. C., Crane, H. D., \& Link, S. W. Effect of display movement on tactile pattern perception. Perception \& Psychophysics, 1966, 1, 195-202.

Bliss, J. C., Crane, H. D., Mansfield, P. K., \& Townsend, J. T. Information available in brief tactile presentations. Perception \& Psychophysics, 1966, 1,.273-283.

Boring, E. G. The two-point limen and the error of localization. American Journal of Psychology, 1930, 42, 446-449.

BorING, E. G. Sensation and perception in the history of experimental psychology. New York: Appleton-Century-Crofts, 1942.

Collins, C. C. Tactile television-mechanical and electrical image projection. Institute of Electrical \& Electronics Engineers Transactions on Man-Machine Systems, 1970, MMS-11, 65-71.

DallenbaCh, K. M. A comparative study of the errors of localization on the fingertips. American Journal of Psychology, 1932, 44, 327-331.

Dinnerstein, A. J., \& Wolfe, M. Tactile slit scanning of letters. Perceptual \& Motor Skills, 1962, 15, 135-138.

Eskildsen, P., Morris, A., Collins, C., \& BaCh-y-Rita, P. Simultaneous and successive cutaneous two-point thresholds for vibration. Psychonomic Science, 1969, 14, 146-147.

Hays, W. L. Statistics for psychologists. New York: Holt, Rinehart, \& Winston, 1963.

HiLL, J. W., \& BuIss, J. C. Modeling a tactile sensory register. Perception \& Psychophysics, 1968, 4, 91-101.

Jenkins, W. L., \& Stone, L. J. Recent research in cutaneous sensitivity: II. Touch and the neural basis of the skin senses. Psychological Bulletin, 1941, 38, 69-91.

Linvill, J. G., \& Bliss, J. C. A direct translation reading aid for the blind. Proceedings of Institute of Electrical \& Electronics Engineers, 1966, 54, 40-51.

SCADDEN, L. A. Perception of patterned vibratory stimulation: An evaluation of the tactile vision substitution system. Doctoral thesis, University of the Pacific, 1971.

TAEnzer, J. C. Some psychophysical limitations on reading performance. Technical Report No. 4828-4, Solid-State Electronics Laboratory, Stanford Electronics Laboratories, Stanford University, 1971 .

TAEnzer, J, C. An information processing model for visual and tactile reading. Perception, 1972, 1, 147-160.

WeINSTEIN, S. Intensive and extensive aspects of tactile sensitivity as a function of body part, sex, and laterality. In $D$. R. Kenshalo (Ed.), The skin senses. Springfield, Ill: Thomas, 1968.

WINER, B. J. Statistical principles in experimental design. New York: McGraw-Hill, 1962.

ZIGLER, M. J. The experimental relation of the two-point limen to the error of localization. Journal of General Psychology, $1935,13,316-331$. 


\section{NOTES}

1. The value of $5.2 \%$ was arrived at in the following way: We assumed that a $S$ had perfect memory of the 13 letters presented in each block and no memory for the letters presented in previous blocks. Thus, the expected number of correct responses for each block is the sum of the expected number of correct responses for each of the 13 trials. Assuming random guessing and sampling without replacement, this sum is $1 / 26+1 / 25+\cdots+1 / 14$. The proportion of correct responses expected on the "average" trial is the above sum divided by 13 .

(Received for publication December 14, 1973; revision received June 17, 1973.)

\section{ERRATUM}

Wade, N. J. Figural effects in afterimage fragmentation. Perception \& Psychophysics, 1974, Vol. 15, No. 1. 115 122. On page 118, Figure 2 was inadvertently omitted. It is presented below with figure caption.

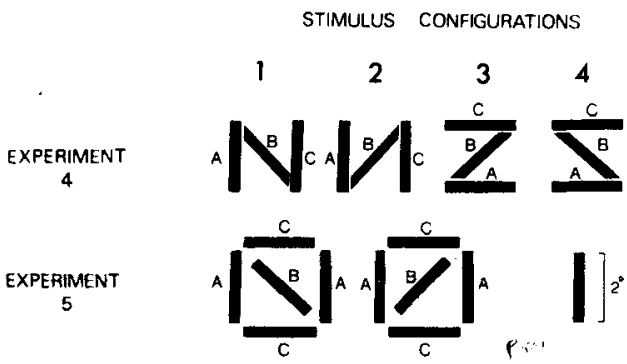

Fig. 2. The stimulus configurations employed in Experiments IV and $V$. 